\title{
Abdominal Pain: A Real Challenge in Novel COVID-19 Infection
}

\author{
Erika Poggiali, Pau Mateo Ramos, Davide Bastoni, Andrea Vercelli, Andrea Magnacavallo \\ ${ }^{1}$ Emergency Department, Guglielmo da Saliceto Hospital, Piacenza, Italy
}

Received: 24/03/2020

Accepted: 25/03/2020

Published: 26/03/2020

How to cite this article: Poggiali E, Mateo Ramos P, Bastoni D, Vercelli A, Magnacavallo A. Abdominal pain: a real challenge in novel COVID-19 infection. EJCRIM 2020;7: doi:10.12890/2020_001632.

Conflicts of Interests: The Authors declare that there are no competing interests.

Acknowledgements: The authors thank all the staff of the Emergency Department of Guglielmo da Saliceto Hospital for their hard work and help.

This article is licensed under a Commons Attribution Non-Commercial 4.0 License

\section{ABSTRACT}

COVID-19 (coronavirus disease 19) is an infectious disease caused by coronavirus 2019-nCoV. Since its detection in China at the end of 2019, the novel coronavirus has rapidly spread throughout the world and has caused an international public health emergency. The most common manifestation is flu-like symptoms. Mild infections usually improve within a few days, but COVID-19 can cause severe pneumonia with acute respiratory distress syndrome and death. Gastrointestinal symptoms are less common but possible and more difficult to recognize as part of a COVID-19 syndrome. In line with the current opinion of the WHO, we strongly believe that preventive measures and early diagnosis of COVID-19 are crucial to interrupt virus spread and avoid local outbreaks. We report the cases of COVID-19 patients admitted to our Emergency Department who complained of gastrointestinal symptoms at admission.

\section{LEARNING POINTS}

- The novel COVID-19 infection is a severe public health problem which is causing an increasing number of deaths worldwide.

- Although uncommon, there may be a relationship between gastrointestinal symptoms and COVID-19, as reported in recent studies.

- Early detection and isolation of patients with COVID-19 infection is the only way to control and limit the global spread of this virus.

\section{KEYWORDS}

COVID-19 infection, abdominal pain, diarrhoea, gastrointestinal symptoms

\section{INTRODUCTION}

The coronaviruses are a large family of viruses that can cause respiratory infections ranging from mild symptoms to severe pneumonia. 2019-nCoV is a new type of coronavirus which was first identified in China. Since its detection in humans at the end of 2019, it has rapidly spread worldwide causing respiratory infections of various degrees of severity. The most common symptoms, which develop within 2-14 days of exposure, are dry cough, fever and fatigue ${ }^{[1,2]}$. Mild infections may be self-limiting in few days, but in some cases the respiratory symptoms can develop into acute respiratory distress syndrome (ARDS) with a high risk of death from multiorgan failure.

Nasopharyngeal and oropharyngeal swabs can be used to confirm the presence of 2019-nCoV. A CT scan of the chest is the gold standard for diagnosis and assessment of lung involvement. Ground-glass opacity and crazy-craving patterning are the most common radiological signs of COVID-19 pneumonia. CT is commonly used to evaluate lung damage during recovery ${ }^{[3]}$. Recently, we investigated the role of lung US in the early detection of COVID-19 pneumonia and showed a strong correlation between lung US and CT scans in all investigated patients ${ }^{[4]}$. No specific treatment or vaccines against COVID-19 are currently available. COVID-19 patients can be treated with antivirals but there is no clear evidence of benefit ${ }^{[5]}$. The most important way to fight this viral infection is to detect COVID-19 early, isolate cases, trace contacts 
and deliver the correct information to the general public ${ }^{[6]}$.

Gastrointestinal involvement, such as abdominal pain, nausea, vomiting and diarrhoea, has been recently reported in the literature ${ }^{[7]}$. Diarrhoea occurs secondary to the interaction between ACE2, highly expressed in the human small intestine, and 2019-nCoV cell entry receptor ACE2. Recent studies show that 2019-nCoV RNA has been detected in stool samples, confirming faecal-oral transmission. According to the literature, the incidence of diarrhoea could be underestimated ${ }^{[8]}$.

In the current case report, we consider COVID-19 infection in the differential diagnosis of gastrointestinal symptoms in 10 patients who referred to our Emergency Department in February 2020, at the beginning of the spread of COVID-19 in Italy.

\section{CASE DESCRIPTION}

Ten patients ( 6 males, 4 females) with mean age 50 \pm 18 years were admitted to our Emergency Department complaining of fever and flulike symptoms in the previous 5-10 days, with general malaise, decreased appetite, abdominal pain and vomiting/diarrhoea. Only one patient denied cough and fever. None of them had a history of abdominal surgery or a notable medical history, including inflammatory bowel disease. They reported no melena or weight loss. Diarrhoea was defined according the WHO criteria as having three or more loose or liquid stools per day or having more stools than normal. Physical examinations were normal with no distended abdomens, tenderness or hypo/ hyperactive bowel sounds. The vital signs were in the normal range with a maximum body temperature of $39^{\circ} \mathrm{C}\left(37.6 \pm 1^{\circ} \mathrm{C}\right)$. At admission, four patients complained of dyspnoea with significantly decreased $\mathrm{PaO}_{2} / \mathrm{FiO}_{2}$ and a need for oxygen.

A nasopharyngeal swab sample confirmed COVID-19 infection in all patients. The patients' characteristics at admission are reported in Table 1.

\begin{tabular}{|c|c|c|c|c|c|c|c|}
\hline & Age (years) & $\operatorname{Sex}(M, F)$ & $\begin{array}{l}\text { Fever and flu-like } \\
\text { symptoms }\end{array}$ & Dyspnoea & GIT symptoms & Body temperature $\left({ }^{\circ} \mathrm{C}\right)$ & $\begin{array}{c}\mathrm{PaO} 2 / \mathrm{FiO} 2 \\
(\mathrm{mmHg})\end{array}$ \\
\hline Pt 1 & 44 & M & No & No & Diarrhoea & 38.7 & $>300$ \\
\hline Pt 2 & 54 & $F$ & Yes & No & Abdominal pain & 39.0 & $>300$ \\
\hline Pt 3 & 39 & $F$ & Yes & No & Diarrhoea & 36.5 & 200 \\
\hline Pt 4 & 44 & M & Yes & Yes & Diarrhoea & 38.8 & $>300$ \\
\hline Pt 5 & 56 & $F$ & Yes & No & Vomiting & 38.5 & $>300$ \\
\hline Pt 6 & 57 & M & Yes & No & Diarrhoea & 36.9 & 275 \\
\hline Pt 7 & 65 & $M$ & Yes & No & Diarrhoea & 37.7 & $>300$ \\
\hline Pt 8 & 22 & $F$ & Yes & Yes & Diarrhoea & 36.8 & 261 \\
\hline Pt 9 & 62 & M & Yes & Yes & Vomiting & 37.8 & 271 \\
\hline Pt 10 & 55 & $M$ & Yes & Yes & Vomiting & 36.0 & 200 \\
\hline
\end{tabular}

Table 1. Patients' characteristics at admission

F, female; GIT, gastrointestinal; M, male; Pt, patient. Abnormal values are in bold.

Laboratory tests revealed lymphopenia, high C-reactive protein and altered liver enzymes in most of the patients, as reported in Table 2. Tests revealed an average white blood cell count (WBC) of $6.15(3.2-9.29) \times 10^{9} /$, platelets (PLT) $176.7(82-341) \times 10^{9} / \mathrm{l}$, and lymphocytes $1.31(0.60-2.76) \times 10^{9} /$ I. The eosinophil percentage was undetectable in all patients. The average value of ALT (alanine aminotransferase) was 77.4 U/I (29-162 U/I), AST (aspartate aminotransferase) was 81.9 (30-284) U/I, and GGT (gamma glutamyltransferase) was 123 (54218) $U /$ l. Bilirubin was increased in only one patient (pt 2, Table 2) who developed pancytopenia and haemolytic anaemia, which improved with oral steroid therapy. The average value of CRP was 6.6 (1.5-21.37) $\mathrm{mg} / \mathrm{dl}$. When measured, procalcitonin was always undetectable, excluding a bacterial infection. Despite the diarrhoea, only two patients had slowly increasing creatinine levels (pt 7 and 9, Table 2). The 
serum potassium level was in the normal range in all patients. In the patient who complained only of diarrhoea with no flu-like symptoms (pt 1, Table 1), we performed stool cultures for Clostridium difficile and enteric pathogens, which were all negative.

All patients were investigated with bedside US, which showed a diffuse B-pattern, due to a severe loss of aeration, with spared areas with no pleural effusion; in six patients subpleural consolidation in the lower lobes was detected. Chest CT confirmed COVID-19 pneumonia with typical imaging signs of ground-glass opacity, patchy consolidation and crazy-paving patterning, which were also detected by lung US. An X-ray of the abdomen was performed in only one patient (pt 2, Table 1) and was normal. US of the abdomen did not reveal an abnormal stomach and bowel distention, except in one female patient (pt 8, Table 1) who had bowel inflammatory signs (peri-intestinal inflammatory reaction) as confirmed by a CT scan of the abdomen. This patient completely recovered after antiviral treatment and was discharged home with no signs of bowel disease on US of the abdomen.

All the patients were treated with supportive care and antiviral therapy, including lopinavir and ritonavir tablets. Diarrhoea completely resolved. Six patients have been discharged with the diagnosis of 'COVID-19 pneumonia and diarrhoea'. Four patients (pt 3, 7, 9, 10, Tables 1 and 2) developed acute severe respiratory distress syndrome requiring immediate intubation. They are still in Intensive Care Unit at the time of writing.

\begin{tabular}{|c|c|c|c|c|c|c|c|c|c|c|}
\hline & Pt 1 & Pt 2 & Pt 3 & Pt 4 & Pt 5 & Pt 6 & Pt 7 & Pt 8 & Pt 9 & Pt 10 \\
\hline WBC $\left(10^{9} / \mathrm{I}\right)$ & 9.20 & 3.40 & 4.03 & 4.91 & 6.24 & 9.85 & 5.40 & 3.20 & 6.04 & 9.29 \\
\hline Neutrophil count $\left(10^{9} / \mathrm{l}\right)$ & 5.73 & 1.40 & 3.21 & 3.28 & 3.68 & 8.61 & 3.54 & 2.39 & 4.46 & 7.43 \\
\hline Lymphocitye count $\left(10^{9} / I\right)$ & 2.76 & 1.69 & 0.60 & 1.08 & 1.99 & 0.94 & 1.32 & 0.66 & 0.86 & 1.20 \\
\hline Eosinophil count $\left(10^{9} / \mathrm{l}\right)$ & 0 & 0 & 0 & 0 & 0 & 0 & 0 & 0 & 0 & 0 \\
\hline PLT count $\left(10^{9} / \mathrm{I}\right)$ & 148 & 82 & 162 & 168 & 143 & 262 & 150 & 165 & 146 & 341 \\
\hline$C R P(<0.5 \mathrm{mg} / \mathrm{dl})$ & 5.3 & 1.54 & 6.15 & 1.5 & 1.19 & 6.53 & 2.55 & 4.09 & 21.37 & 15.85 \\
\hline$P C T(>0.5 \mathrm{ng} / \mathrm{ml})$ & ND & 0.03 & ND & ND & ND & ND & 0.36 & 0.02 & ND & 0.32 \\
\hline AST (10-37 U/I) & 111 & 35 & 82 & 29 & 45 & 162 & 47 & 95 & 86 & 82 \\
\hline ALT $(10-37 \mathrm{U} / \mathrm{I})$ & 101 & 53 & 60 & 30 & 46 & 284 & 57 & 67 & 68 & 53 \\
\hline GGT (7-40 U/I) & 138 & 99 & 133 & ND & ND & 54 & 96 & 175 & 71 & 218 \\
\hline Total bilirubin (0.1-1.1 mg/dl) & 0.84 & 1.33 & 0.63 & 0.53 & 0.47 & 0.79 & 0.60 & 0.58 & 0.71 & 0.95 \\
\hline LDH $(0-248 \mathrm{U} / \mathrm{I})$ & 400 & 223 & 634 & 251 & 254 & 343 & 244 & 453 & 594 & 964 \\
\hline Lipase (7-60 U/I) & 133 & 22 & 61 & 42 & 42 & 22 & 24 & 169 & 26 & 175 \\
\hline Creatinine $(0.6-1.2 \mathrm{mg} / \mathrm{dll})$ & 1.10 & 0.41 & 0.68 & 1.04 & 0.59 & 0.94 & 1.20 & 0.72 & 1.25 & 1.18 \\
\hline Blood urea nitrogen $(10-50 \mathrm{mg} / \mathrm{dl})$ & 33 & 19 & 28 & ND & ND & ND & 36 & 21 & 42 & 43 \\
\hline Potassium $(3.5-5.0 \mathrm{mEq} / \mathrm{l})$ & 4.3 & 4.0 & 3.8 & 3.5 & 3.7 & 3.7 & 4.3 & 3.8 & 4.4 & 4.4 \\
\hline
\end{tabular}

Table 2 Laboratory findings at admission

ALT, alanine transaminase; AST, aspartate transaminase; CRP, C-reactive protein; GGT, gamma glutamyltransferase; ND, not done; PCT, procalcitonin; PLT, platelet; WBC, white blood cell count. Normal range values are in brackets. Abnormal values are in bold 


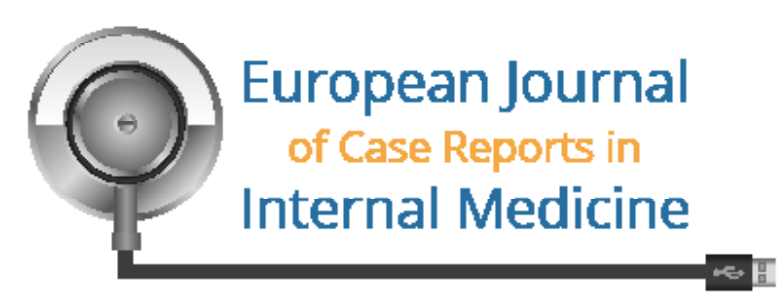

\section{DISCUSSION}

Abdominal pain is a real challenge for critical care physicians since there are many potential diagnoses. Signs and symptoms are often nonspecific and can mask life-threating conditions. The consequences of wrongly attributing the pain can be serious for patients. Although gastrointestinal symptoms are less common in patients with COVID-19 infection, the diagnosis of coronavirus disease 2019 cannot be excluded and must be investigated in all patients referred to the Emergency Department. We strongly recommend bedside lung US to detect signs of interstitial pneumonia even in absence of respiratory symptoms. Early suspicion and diagnosis are essential to contain the global spread of COVID-19 infection and its potentially fatal complications, such as acute respiratory distress syndrome.

\section{REFERENCES}

1. Chen N, Zhou M, Dong X, Qu J, Gong F, Han Y, et al. Epidemiological and clinical characteristics of 99 cases of 2019 novel coronavirus pneumonia in Wuhan, China: a descriptive study. Lancet 2020;395(10223):507-513

2. Huang C, Wang Y, Li X, Ren L, Zhao J, Hu Y, et al. Clinical features of patients infected with 2019 novel coronavirus in Wuhan, China. Lancet 2020;395(10223):497-506.

3. Feng P, Tianhe Y, Peng S, Shan G, Bo L, Lingli L, et al. The course of lung changes on chest CT during recovery from 2019 novel coronavirus (COVID-19) pneumonia. Radiology 2020 Feb 13:200370. doi: 10.1148/radiol.2020200370.

4. Poggiali E, Dacrema A, Bastoni D, Tinelli V, Demichele E, Mateo Ramos P, et al. Can lung ultrasound help critical care clinicians in the early diagnosis of novel coronavirus (COVID-19) pneumonia? Radiology 2020 Mar 13:200847. doi: 10.1148/radiol.2020200847.

5. Cao B, Wang Y, Wen D, Liu W, Wang J, Fan G, et al. A trial of lopinavir-ritonavir in adults hospitalized with severe Covid-19. N Engl J Med 2020 Mar 18. doi: 10.1056/ NEJMoa2001282.

6. World Health Organization. Statement on the second meeting of the International Health Regulations (2005) Emergency Committee regarding the outbreak of novel coronavirus (2019-nCoV). Available from: https://www.who.int/news-room/detail/30-01-2020-statement-on-the-second-meeting-of-the-international-health-regulations(2005)-emergency-committee-regarding-the-outbreak-of-novel-coronavirus-(2019-ncov). Accessed 14 February 2020.

7. Song Y, Liu P, Shi XL, Chu YL, Zhang J, Xia J, et al. SARS-CoV-2 induced diarrhoea as onset symptom in patient with COVID-19. Gut 2020 Mar 5. pii: gutjnl-2020-320891. doi: 10.1136/gutjnl-2020-320891.

8. Liang W, Feng Z, Rao S, Xiao C, Xue X, Lin Z, et al. Diarrhoea may be underestimated: a missing link in 2019 novel coronavirus. Gut 2020 Feb 26. pii: gutjnl-2020-320832. doi: 10.1136/gutjnl-2020-320832. 